\title{
Comparazione tra nativi digitali e adulti nell'approccio alla "rete": analisi delle differenze e del rischio di IAD Una indagine ASL Milano 1 - CS\&L
}

\author{
Fabio Guerrini ${ }^{1}$, Liliana Formenti², Paola Duregon ${ }^{3}$, Moira Fontana ${ }^{4}$, Marco Forlani ${ }^{5}$, \\ Lorenzo Canafoglia ${ }^{6}$, Katia Salemi 7 , Antonio Bellicoso ${ }^{8}$, Rosa Lavilla ${ }^{9}$, Lorella Vignatio $^{10}$
}

- There are many studies and researches dedicated to the IAD from 15-20 years approximately. Nowadays it's so easy to surf the Internet that the number of Internet users has increased, especially among teenagers (also called digital natives); that's why in this generation it's easy to think that the risk of developing a IAD is higher than before.

A survey can show us this problem: a group of under 18 students and a group of adults have done a test (checked by dr K. Young) about how much they are used to surf the net to establish their risk of IAD (which can be classified as a low, medium or high profile). Results show that 34,5\% of under 18 has a medium profile while among the adults the percentage is of $4.20 \%$. For this reason it seems that teenagers are closer to IAD than the adults. However the high risk is of $1.10 \%$ among the under 18 and of $0.84 \%$ among the adults which is a not statistically significant difference. So we can make another hypothesis: some teenagers achieve a medium profile but it doesn't evolve into a high profile of IAD as if there were coltural "antibodies" against the problem. To find a policy against the IAD it's quite important to choose to believe to the first or to the second hypothesis: in fact they have different ways to act. Believing to the first one, we should spend researches for the selective and the indicated prevention. Believing to the second one it's better to focus on informations, advertisements on tablets and smartphones and advices for a correct use. It's recommended to do other researches to find out more demonstrations to those theories.

Keywords: IAD (Internet Addiction Disorder), Digital Natives, Internet Addiction Test, New Addictions, Health Promotion Programs.

Parole chiave: Disturbo/Dipendenza da Internet, Nativi Digitali, Test Dipendenza Internet, Dipendenze Comportamentali, Programmi di Promozione alla Salute.

\section{Introduzione}

Nel corso degli ultimi anni grande attenzione è stata dedicata alle Dipendenze "comportamentali", il cui correlato neurofisiopatologico è risultato essere affine a quello delle più note sostanze psicoattive.

Il clamore suscitato dalle problematiche legate al Gioco Patologico ha impressionato significativamente l'opinione pubblica e la presenza di una offerta di "giochi" molto più ampia del passato incoraggia larghe fasce della popolazione ad accedervi,

\footnotetext{
1. Dirigente medico referente Ser.T. Corsico.

2. Infermiera Sert Corsico.

3. A.soc. coordinatrice Progetti Dipart. Dip. ASL MII.

4. Dirigente psicologo NOA Baranzate.

5. Responsabile Area Lavoro CS\&L.

6. E.p. coordinatore Progetto, $C S \& L$.

7. Responsabile Progetto, $C S \& L$.

8. A.soc. NOA Legnano.

9. E.p. Ser.T. Magenta.

10. A.soc. Ser.T. Parabiago.
}

amplia la numerosità dei Giocatori "sociali" e quelle dei giocatori "problematici" e "patologici".

La complessità degli aspetti clinici, sociali e legali incute grande preoccupazione per le risorse umane, materiali e finanziarie da dedicare alla cura ed alla riabilitazione.

Non meno preoccupante per la risonanza dei dati pubblicati è la situazione correlata alla tematica dei Disturbi da eccessivo utilizzo delle tecnologie digitali (Internet Addiction Disorder o IAD).

Essi insorgono quando un utilizzo ludico-ricreativo, (che si potrebbe definirebbe "sociale") lascia il posto a quadri clinici ben classificati ad hoc da Goldberg già nel 1995.

Egli ne evidenziò la capacità di indurre tolleranza (necessità di aumentare il tempo di connessione per soddisfare un livello di gratificazione sufficiente) ed astinenza (da qualche giorno a un mese dopo la "disconnessione" dalla rete) con ripercussioni negative nella vita dell'individuo che si riverberano negli ambiti relazionale e familiare: isolamento, ritiro sociale, peggioramento delle performances lavorativa e scolastica causate dal tempo prioritariamente dedicato alla connessione informatica, ma anche alterazioni dello stile di vita, tendente alla sedentarietà, disturbi "somatici" (dorsalgie, cefalee, fastidi oculari, insonnia) 
nonché problemi in ambito finanziario quando il Disturbo si concentra in scommesse on line, shopping on line, ecc.

Quest'ultimo aspetto, poi, rende lo IAD trasversale anche ad altre Dipendenze comportamentali: non solo Gioco Patologico (o Net gaming) e Shopping compulsivo ma anche Sexual Addiction (o ciber sessuale) ampliando, quindi, le difficoltà terapeutiche di fronte a Polidipendenze Comportamentali che hanno verosimili aspetti comuni ma anche peculiarità e specificità esigenti approcci diversificati.

Negli adolescenti i Disturbi assimilabili all'IAD sono spesso inquadrati in una sottocategoria nosologica tipica della giovane età: la dipendenza ciber-relazionale, o da Social Networks (per esempio Facebook, FB) nella quale si arriva a preferire le relazioni "virtuali" a quelle reali tanto che lo psicologo inglese D. Smallwood (2008) coniò il termine di friendship addiction per la elevata frequenza con la quale gli utenti di FB collezionano il maggior numero di amici sul proprio profilo oppure su nuovi profili, questi ultimi quasi delle identità alternative mediante le quali diversificare ed assegnare i numerosi amici contattati.

Spesso i nuovi profili (talora anche profili fake), sono omonimi di colui che li ha creati ma, ovviamente, più "performanti" e accattivanti.

A questa iniziale situazione "artificiale" ed inebriante della ricerca continua di nuovi amici fa seguito, soprattutto se nella vita reale emergono difficoltà nelle relazioni con controparti "in carne e ossa", una fase di intensificazione della connessione a FB (il controllo continuo dello "stato", dei post e dei "mi piace") alla ricerca non solo di nuovi contatti ma, in primis, di una gratificazione che nelle relazioni reali sembra mancare.

Ad uno stadio ulteriore la friendship addicton diviene pervasiva, si incomincia a non distinguere più nitidamente il confine tra la propria identità privata e quella pubblica della "rete" e ne risentono ancora i rapporti reali e la gestione dell'emotività nella vita di relazione vera (F. Saccà-Roma), nella quale persino lo stile comunicativo verbale di FB diventa predominante. A questo punto, come in altre dipendenze, l'impulso a "collegarsi" (inizialmente finalizzato alla sola gratificazione) si confonde con la compulsività (collegarsi diventa necessario per attenuare una sensazione pervasiva di disagio), I'isolamento sociale prende il sopravvento e, talora, possono comparire idee persecutorie ( la "rete" complotta contro il soggetto) con ansia associata fino a pensieri rimuginanti e paranoidi. È evidente, a questo stadio, la necessità di un percorso diagnostico e di cura.

\section{Contesto culturale e scopo della indagine dell'ASL Milano 1}

Nell'attuale epoca è oramai fisiologico "collegarsi" e sfruttare i vantaggi innumerevoli offerti dalla "rete", quotidianamente, a domicilio o in mobilità, per cui il numero degli utenti è elevatissimo, in particolare tra i giovanissimi o "nativi digitali".

Nel confronto con le generazioni adulte, infatti, essi hanno acquisito l'uso del web in età prepuberale (ovvero in periodo di grande "plasticità" cerebrale), spesso per fini ricreativi e socializzanti quindi non solo scolastico-culturali.

Questa differenza generazionale è, apparentemente, una ovvietà tuttavia è utile approfondirla, provare a quantificarla, non accontentandosi solo di intuirne e constatarne l'entità.

Questa indagine si propone, infatti, di misurare le differenze tra adulti ed under 18, di cercare il punto di partenza dal quale muovere per parlare veramente di rischio di IAD come se si dovesse stabilire il punto al quale posizionare una asticella per una competizione di salto in alto: va da sé che si debba rapportare il primo punto di posizionamento con il livello degli atleti (principianti o campioni).

Fuor di metafora, infatti, è chiaro che di scarsa utilità sarebbe ipotizzare che il punto di partenza per gli adolescenti sia pari a quello degli adulti: facile sarebbe concludere che larga parte dei teen agers sia a rischio di IAD sottostimando che le maggiori assiduità e continuità di interazione con la "rete" da parte dei giovanissimi sono l'esito di un contesto culturale diverso da quello degli adulti.

Solo in un secondo momento, reperito il loro preciso punto di partenza, è sensato valutare se le abitudini degli adolescenti rivelino un uso più evoluto e più consapevole della "rete" oppure un rischio verosimile di avvicinamento ad un disturbo additivo.

Al fine di chiarire tali riflessioni, il presente lavoro procede con un'analisi dei risultati ottenuti da un test rivolto sia ad un gruppo di studenti sia ad un gruppo di adulti in merito alle loro abitudini di frequentazione del web.

Si vedrà che l'esito della comparazione è riconducibile ad una "tendenza" ampiamente attesa ed in linea con il contesto culturale attuale, e sembra indicare, per i giovanissimi, un rischio di prognosi sfavorevole più elevato di quello degli adulti, suggerendo futuri interventi di prevenzione selettiva o indicata.

Tuttavia I'analisi più approfondita dei risultati permette di formulare una ipotesi alternativa che promuove, in vece, la destinazione di eventuali risorse ad interventi di prevenzione universale.

A conferma della diffusione del fenomeno e della necessità di intraprendere azioni preventive di "massa" si riporta la proposta di alcuni ricercatori inglesi (Raian et al., 2015) sintetizzata nella applicazione incondizionata di etichette su tablet e smartphone con avvisi sui pericoli correlati all'uso senza limite delle tecnologie digitali come accade per le sigarette.

\section{Materiali, metodi, gruppi analizzati}

La ricerca origina all'interno del progetto "Salute e Lavoro", organizzato dal Dipartimento Dipendenze della ASL Milano 1 in partnership con il Consorzio CS\&L, un progetto di promozione alla Salute nei luoghi di lavoro, di prevenzione dei comportamenti "additivi" ed il cui fine è di sensibilizzare i lavoratori rispetto all'uso, anche occasionale, di sostanze (alcool, farmaci, THC) che per la loro interferenza con l'ambito professionale potrebbero causare infortuni sul lavoro o incidenti correlati (per esempio per l'assunzione di alcool durante la pausa pranzo o prima dei turni lavorativi).

Fin dal 2007 il Progetto si è articolato in numerosi interventi nelle aziende del territorio: pianificazione condivisa, questionari, corsi di formazione, valutazione e monitoraggio a distanza della ricaduta delle azioni intraprese, anche nell'auspicio della "moltiplicazione" di una corretta informazione nella comunità territoriale (inoltre confutando "false credenze" e "luoghi comuni" sulle sostanze psicoattive).

Esso è stato inserito nella banca dati dell'Osservatorio sulle Dipendenze di Lisbona come da scheda EDDRA (Excange on Drug Demand Reduction Action) in quanto progetto di interesse europeo in particolare per l'aspetto della valutazione degli esiti degli interventi svolti.

Il Progetto prosegue tuttora in alcune aziende del territorio delI'ASL. 
Fig. 1

\begin{tabular}{|c|c|c|}
\hline & Affermazione (item) & Risposte \\
\hline 1 & Preferisco stabilire rapporti on line piuttosto che uscire con gli amici & mai, ogni tanto, sempre \\
\hline 2 & Mi capita spesso di alzare la voce se qualcuno mi disturba mentre sono collegato & mai, ogni tanto, sempre \\
\hline 3 & Se non uso internet per un giorno intero mi sento un po' "scoppiato" (agitato) & mai, ogni tanto, sempre \\
\hline 4 & $\begin{array}{l}\text { Penso che la vita sia noiosa e vuota senza internet; } \\
\text { sto benissimo quando sono sempre collegato/a }\end{array}$ & mai, ogni tanto, sempre \\
\hline 5 & $\begin{array}{l}\text { Cerco di nascondere il tempo che passo on line. Dico "ancora qualche minuto } \\
\text { e spengo", ho provato a ridurre il tempo che passo collegato, ma non ci sono } \\
\text { riuscito }\end{array}$ & mai, ogni tanto, sempre \\
\hline 6 & $\begin{array}{l}\text { Non vedo l'ora di collegarmi a internet; mi collegherei ovunque perché spesso } \\
\text { la mia tranquillità è associata alla possibilità di connettermi. }\end{array}$ & mai, ogni tanto, sempre \\
\hline 7 & $\begin{array}{l}\text { Spesso i miei familiari e colleghi si lamentano perché sono sempre collegata } \\
\text { a internet, trascuro quello che devo fare e perdo anche ore di sonno. }\end{array}$ & mai, ogni tanto. spesso \\
\hline
\end{tabular}

Una declinazione progettuale è stata l'organizzazione di eventi, denominati "Mind Trick", che prevedevano, in ambienti allestiti ed arredati ad hoc, I'interazione dei lavoratori con supporti audiovisivi e postazioni informatiche, presso le quali essi si cimentavano in giochi e test all'interno di un percorso "a tappe" atto a promuovere riflessioni relative ai rischi correlati alle Dipendenze e a diffondere informazioni da spendere anche in altri ambiti di vita (in quanto genitori, tecnici sportivi, catechisti, ecc.).

Tra le varie "tappe" proposte ne è stata organizzata una dedicata alla abitudine di collegarsi al world wide web: essa proponeva all'interlocutore (collegato singolarmente ad un PC) la visione e l'ascolto di un cortometraggio di fattura "amatoriale" munito di scenografia e accompagnamento musicale nel quale diversi attori comparivano in successione recitando una breve affermazione (assimilabile ad un item) legata all'abitudine di connettersi alla "rete informatica" (vedi fig. 1).

Allo spettatore veniva lasciato il tempo di compilare una scheda dove, per ognuna delle 7 affermazioni proposte, egli barrava la risposta più vicina alla propria situazione.

Al termine delle 7 scene filmate, la voce guida del cortometraggio indicava di conteggiare il numero delle risposte "mai, ogni tanto, sempre" ed, in base alla frequenza, assegnava al partecipante un profilo tra i 3 possibili (fig. 2).

In base al profilo ottenuto la "voce guida" del cortometraggio forniva suggerimenti ed eventuali proposte correttive. Veniva offerto anche di prelevare materiale cartaceo al riguardo.

Fig. 2

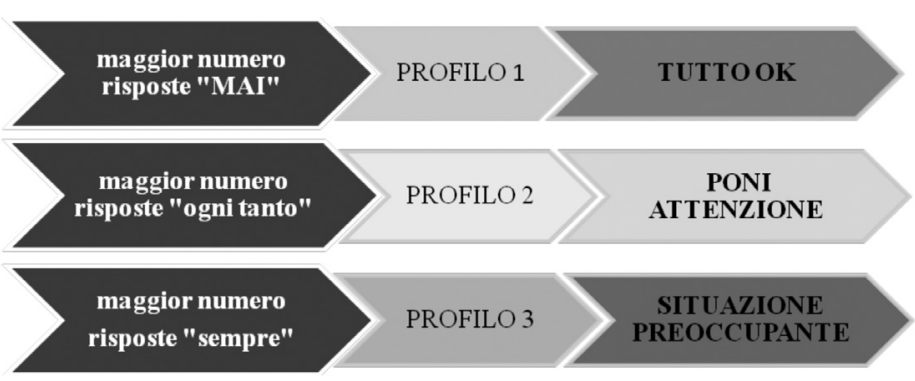

L'evento Mind Trick ha registrato buoni apprezzamenti, per cui è stato riproposto in altri contesti (centri commerciali, centri culturali comunali) infine è stato richiesto da una scuola frequentata da alunni di 14-16 anni.

L'attualità dell'argomento nonché gli strumenti ludici utilizzati ben si conciliavano anche con tale target.

La disponibilità dei dati raccolti nella scuola e nell'azienda (poste nel medesimo territorio) mediante il medesimo format di Mind Trick ha promosso una comparazione dei risultati ottenuti da 119 lavoratori di vari profili professionali tra i 30 e i 50 anni da un lato (Gruppo 2) e 90 alunni tra i 14 ed i 16 anni dall'altro (Gruppo 1).

L'analisi ha compreso: il confronto statistico dei profili di rischio ottenuti; il calcolo della frequenza delle 3 risposte possibili ("mai","ogni tanto", "sempre") e la loro distribuzione nei 2 gruppi, ma anche la valutazione di alcuni singoli items scelti quali indicatori di rischio "additivo". Il test statistico prescelto è stato quello del "confronto tra proporzioni" integrato a quello del "chi quadrato" svolti mediante software applicativo di Stanton Glantz, Statistica per discipline biomediche (Ed. McGraw-Hill, Edizione quarta).

Il valore di significatività è stato posto a $P<0,005$.

Il questionario proposto fa riferimento a quello della dr.ssa $\mathrm{K}$. Young (Center for Internet Addiction Recovery di Pittsburgh) con alcune modifiche: nella versione originale si compone di 20 quesiti e cinque risposte (mai, raramente, ogni tanto, spesso, sempre) con punteggi abbinati ad ogni risposta (da 1 a 5 punti), richiede circa 10 minuti per lo svolgimento ed il punteggio finale assegna uno tra 3 profili possibili (utente normale, utente a rischio, utente problematico).

Nella nostra indagine, dovendo conciliare esigenze di vario genere (tempi di esecuzione del test non superiore a 3/4 minuti, presenza di una singola postazione informatica, brevità del cortometraggio), sono state scelti 7 item tra i 20 del test americano, giudicati significativi e sufficienti a rivelare abitudini "improprie". Analogamente al test della dr.ssa Young le risposte sono state ordinate secondo scala nominale (mai, ogni tanto, sempre) eliminando le voci "raramente" e "spesso" ma identificando, comunque, i medesimi 3 profili di rischio. 


\section{Risultati e ipotesi correlate}

Come visibile dal diagramma circolare (fig. 3), il gruppo degli adulti si è posizionato in grande maggioranza $(89,92 \%)$ nel profilo 1 (utente normale) in minor misura $(4,2 \%)$ nel profilo 2 (utente a rischio), infine in misura piccolissima $(0,84 \%)$ nel profilo 3 (uso improprio della rete).

Fig. 3

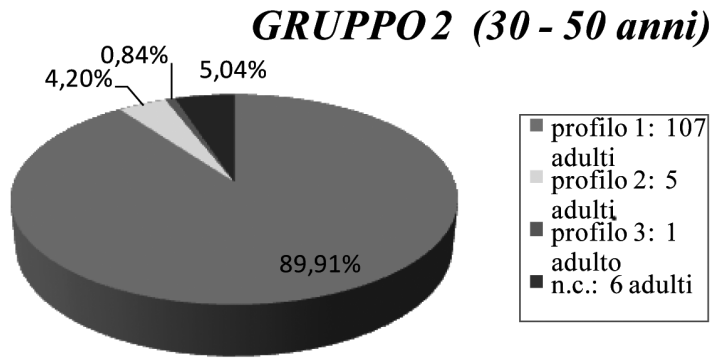

Diversamente, nel gruppo degli adolescenti il profilo 1, benché maggiormente rappresentato $(58,9 \%)$, è controbilanciato da una significativa presenza del profilo $2(34,5 \%)$, infine il profilo 3 è, anche in questo caso, il meno rappresentato $(1,10 \%)$ (fig. 4$)$.

Fig. 4

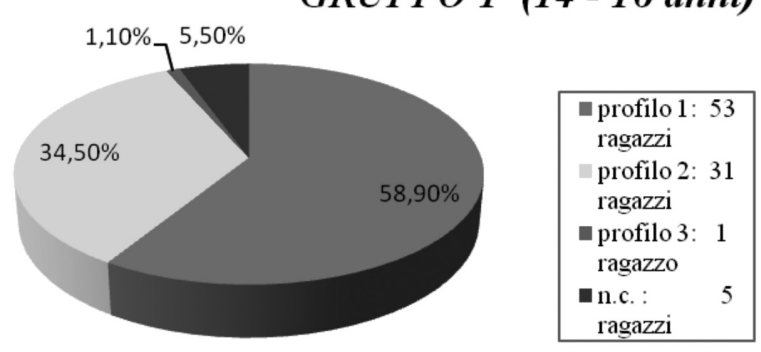

Confrontando le proporzioni dei profili (Profilo 1 adulti versus profilo 1 studenti, profilo 2 e 3 adulti rispettivamente versus gli omologhi degli studenti) mediante il metodo del confronto di proporzioni si ottengono dei valori di $p<0.001$ per quanto riguarda il profilo 1 ed il profilo 2 .

Nessuna significatività, in vece, per la comparazione del profilo 3 ovvero quello di maggior gravità.

A verifica del dato acquisito sono state comparate le percentuale di risposte, ignorando, in questo caso, i profili assegnati: gli adulti hanno scelto l'80\% di risposte "mai", il 15\% di risposte "ogni tanto" e l'1,32\% di "sempre"; i ragazzi il 53,8\% di mai, il $40 \%$ di "ogni tanto" ed il 4,7\% di "sempre".

Utilizzando il test del "Chi quadrato" (per 3 gradi di libertà) si è ottenuto un valore di significatività statistica consistente $(p<0.001)$.

Poiché lo scopo dell'indagine era quello non solo di comparare le risposte degli adolescenti con quelle degli adulti, ma anche verificare la presenza di "sintomi" da sovraesposizione al $w w w$ è stata condotta un'analisi di alcune singole risposte, ritenute "spie" di comportamenti potenzialmente problematici,

Nelle figure 5 e 6 si osserva la loro distribuzione nei 2 gruppi.

All'item n. 5 " Cerco di nascondere il tempo che passo on line. Dico: ancora qualche minuto e spengo, ho provato a ridurre il tempo che passo collegato, ma non ci sono riuscito") si nota che il $56,6 \%$ dei ragazzi si ritrova nella situazione proposta "ogni tanto" (52,2\%) o addirittura "sempre" (4,4\%).
Fig. 5

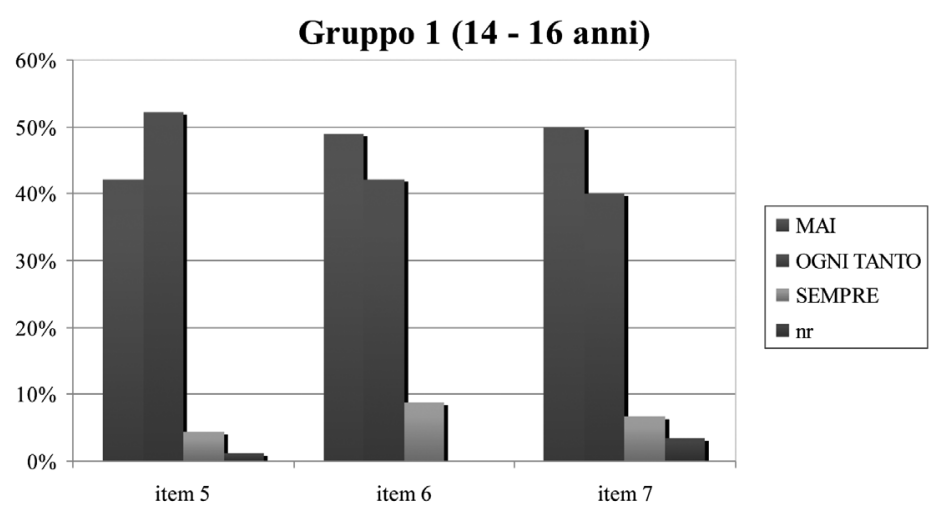

Fig. 6

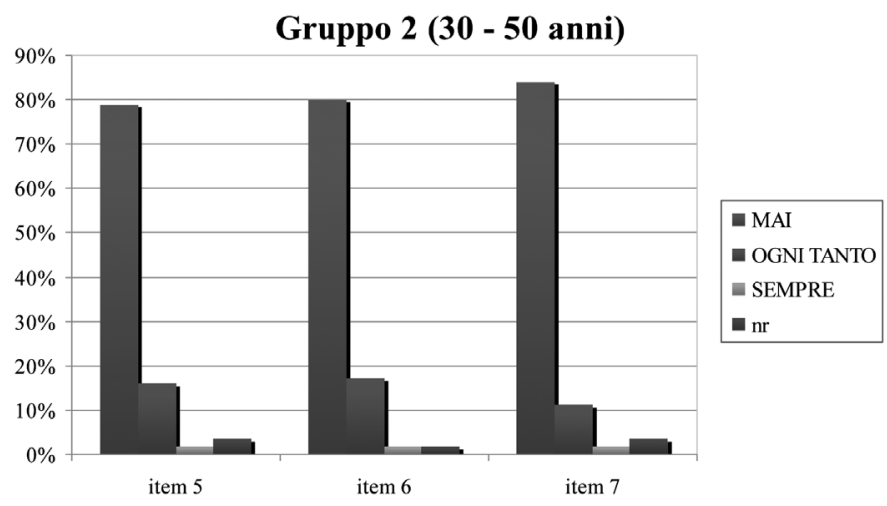

Ma anche all'item 6 ("Non vedo l'ora di collegarmi a internet; mi collegherei ovunque perché spesso la mia tranquillità è associata alla possibilità di connettermi") il 51\% degli studenti ha trovato corrispondenza con la propria situazione personale, il $42,2 \%$ ogni tanto, $I^{\prime} 8.8 \%$ sempre.

Dalla figura 6 si può notare, invece, che gli adulti non hanno mai raggiunto il $20 \%$ di risposte "ogni tanto" o "sempre" in nessuno dei 3 items analizzati a conferma che il cambio generazionale, in tema di approccio alla "rete", è di proporzioni significative (e non solo dal punto di vista statistico).

Come già rilevato, i profili ottenuti dagli studenti comprendono una esiguità di Profili 3 (rischio concreto), ovvero I'1,1\%, dato che autorizza a pensare ad una situazione complessivamente accettabile e scarsamente preoccupante anche per l'assenza di significatività statistica nella comparazione con gli adulti di pari profilo $(0,84 \%)$.

Tuttavia, se si ragionasse sul "peso" del Profilo 2 (rischio iniziale), raggiunto dal $34,5 \%$ dei teen agers (laddove gli adulti si attestano al 4,2\%), unitamente ai dati emersi dagli items 5 e 6 , sarebbe naturale sospettare un diffuso segno di sovraesposizione al mezzo informatico (quindi non una innocua abitudine) e, quindi, temere che una parte di Profili 2, nel breve o medio termine - vista la giovane età degli intervistati - possa evolvere al Profilo peggiore.

Oltretutto i ragazzi dell'indagine sembrano ben consapevoli della situazione: il dato dell'item 7 ("Spesso i miei familiari e colleghi si lamentano perché sono sempre collegato a internet, trascuro quello che devo fare e perdo anche ore di sonno") lo confermerebbe ampiamente (le risposte "ogni tanto" e "sempre" raggiungono il $46,6 \%$ ). 


\section{Discussione}

I Disturbi da uso improprio ed eccessivo della "rete" (meglio definiti come Internet Addiction Disorder) sono oggetto di attenzione oramai da circa 15-20 anni.

L'abitudine a collegarsi ad essa è senza dubbio segnale di progresso per i numerosi vantaggi diffusamente distribuiti nelle più disparate aree della vita umana. Tuttavia, come già osservato nel GAP, la maggior disponibilità dell'"oggetto" ricreativo può far emergere, in soggetti con latente vulnerabilità individuale, quadri clinici patologici ascrivibili ad una addiction.

Nel caso dell'interazione con le reti informatiche le generazioni più giovani ("nativi digitali") sono cresciute con un più agevole accesso al web (praticamente dovunque e sempre), in particolare nei confronti dei vari social network.

È da capire, però, se gli adolescenti attuali utilizzino la rete con modalità evolute e consapevoli rispetto agli adulti (che vi si sono avvicinati a maturità cerebrale acquisita e a stili di vita consolidati), oppure se siano esposti a maggiori potenzialità di rischio "additivo".

Questa indagine, frutto di una interazione tra ASL e CS\&L, utilizza i dati raccolti da due gruppi (adulti in età lavorative versus adolescenti di 14-16 anni appartenenti al medesimo ambito territoriale), allo scopo di misurare le loro differenze (e conseguenze) nella interazione con la "rete". Se da un lato, infatti, è facile attendersi che gli adolescenti siano utenti di Internet degli adul$\mathrm{ti}$, il problema è capire, anche in un'ottica previsionale, se il riscontro di numerosi Profili 2 (34.5\% contro il 4,2\% degli adulti) misurati in questo gruppo nasconda un ampio e diffuso rischio futuro di evoluzione verso il Profilo peggiore (Profilo 3) a meno di una rimodulazione della abitudine a "collegarsi".

In effetti I'analisi parcellare del test (item 5-6-7) sembra supportare I'ipotesi di un futuribile peggioramento poiché una larga parte dei nostri adolescenti ci dice (tra il 45 ed il 55\%) che, almeno saltuariamente, fatica a disconnettersi benché provi a farlo; giudica la connessione altamente gratificante e prioritaria per arrivare ad una sensazione di tranquillità soddisfacente; è consapevole che tale comportamento desta insofferenza e preoccupazione altrui.

Per contro gli adulti che hanno risposto analogamente agli adolescenti a questi item non oltrepassano mai la soglia del $20 \%$ ed il profilo 2 viene raggiunto solo dal 4,2\% del loro gruppo, espressione verosimile di un contesto di "nicchia".

Si dovrebbe, quindi, concludere, per la rappresentatività del profilo 2 e per i segnali emersi dai 3 item analizzati, che i teen agers siano a rischio addiction elevato rispetto agli adulti, temendo un futuribile loro posizionamento in profili di rischio più gravi, elemento che solleciterebbe I'adozione di interventi correttivi specifici.

È possibile, però, anche una "lettura" alternativa dei risultati, correlata al dato dei profili "gravi" riscontrati nella nostra indagine (profilo 3), bassissimo negli adolescenti -1,1\% - così come negli adulti, 0,8\%. Focalizzando l'attenzione su questo dato si potrebbe pensare che per gli adolescenti il punto di partenza per catalogare il rischio di IAD sia molto diverso da quello del mondo adulto. Infatti, se a fronte di un elevato numero di under 18 posizionato all'interno del profilo $2(34,5 \%)$ fa riscontro un numero così contenuto di utenti problematici (profilo $3-1,1 \%$ ), sarebbe realistico ipotizzare che il contesto culturale in cui essi sono cresciuti potrebbe aver promosso in molti di loro una sorta di "immunità comportamentale" che limita un futuribile peggioramento lasciandoli in una situazione semplicemente da sorvegliare nel tempo.

L'antitesi tra le due possibili conclusioni - ovvero che i "nativi digitali" siano ampiamente a rischio di IAD oppure, al contrario, che sia "fisiologico" ritrovarne molti potenzialmente a rischio (profilo 2), ma pochissimi davvero problematici (profilo 3) non appare questione banale in un'ottica di politiche sociali e di investimenti, poiché nel primo caso andrebbero promossi interventi di prevenzione indicata e selettiva, nel secondo sarebbe preferibile intervenire con iniziative di prevenzione primaria e "universali" (decaloghi di comportamento corretto, disponibilità ampia di tests di autovalutazione nelle scuole, avvisi sulle confezioni di tablet e smartphones, ecc.).

Questa indagine potrebbe essere, quindi, un verosimile punto di partenza dal quale sviluppare eventuali conferme o smentite e aumentare I'attenzione dedicata al tema dell' IAD da parte degli operatori dei Ser.t. e non solo dei ricercatori.

\section{Bibliografia}

Kimberly S. Young (2000), Presi nella rete. Intossicazione e dipendenza da Internet, Calderoni Edizioni (a cura di T. Cantelmi).

Kimberly S. Young (1996), "The Emergence of a New Clinical Disorder", Cyberpsycology and Behavior, vol. 1, n. 3: 237-244.

Raian Ali et al. (2015), The Emergency Requirement for Digital Addiction Labels, Bournemouth University UK, www.staffprofiles.bourneamouth.ac.uk. Smallwood D. (2008), Articoli tratti da www.davidsmallwoodtherapy.co.uk. Saccà F. (2012), Articoli tratti da www.francescasacca.it.

È vero che esiste la dipendenza da Internet?, a cura di dr. F. Tonioni (Policlinico Gemelli - Roma), Articolo tratto da www.fondazioneveronesi.it (2013). 